hep-ph/0302073

\title{
Flavor Hierarchy in SO(10) Grand Unified Theories via 5-Dimensional Wave-Function Localization
}

\author{
Ryuichiro Kitano* and Tianjun $\mathrm{Li}^{\dagger}$ \\ School of Natural Sciences, Institute for Advanced Study, Princeton, NJ 08540
}

September 29, 2018

\begin{abstract}
A mechanism to generate fermion-mass hierarchy in $\mathrm{SO}(10)$ Grand Unified Theories is considered. We find that the lopsided family structure, which is suitable to the Large angle MSW solution to the solar neutrino oscillation, is realized without introducing extra matter fields if the hierarchy originates from the wave-function profile in an extra dimension. Unlike the Froggatt-Nielsen mechanism, the SO(10) breaking effect may directly contribute to the source of the hierarchy, i.e., the bulk mass terms. It naturally explains the difference of the hierarchical patterns between the quark and the lepton sectors. We also find the possibility of the horizontal unification, in which three generations of the matter fields are unified to a $\mathbf{3}$ dimensional representation of an $\mathrm{SU}(2)$ gauge group.
\end{abstract}

*email: kitano@ias.edu

$\dagger$ email: tli@sns.ias.edu 


\section{Introduction}

Supersymmetric Grand Unified Theory (SUSY GUT) is one of the most promising candidates which describe the interactions among elementary particles in very high energy phenomena [1]. SUSY extension of the Standard Model provides us a solution to the hierarchy problem [2], and the unification of three gauge interactions in the Standard Model to GUT symmetries explains the charge quantization which is realized in nature [3]. Moreover, these two scenarios are non-trivially consistent with each other since SUSY predicts the coincidence of the gauge coupling constants at the high energy scale [4, which is a necessary condition to realize the Grand Unification.

Among several candidates for the GUT gauge group such as $\mathrm{SU}(5), \mathrm{SO}(10)$, and $\mathrm{E}_{6}$, $\mathrm{SO}(10)$ has particularly attractive features. One is the matter unification. All the quarks and leptons including right-handed neutrinos in each generation are unified to a single 16 dimensional spinor representation field. Also, $\mathrm{SO}(10)$ is the smallest simple GUT group which does not require a particular particle content to cancel the gauge anomaly. Yukawa unification is another interesting point. Because of the matter unification to 16 representation fields, the Yukawa-interaction terms between the matter $\left(\mathbf{1 6}_{i}\right)$ and the Higgs $\left(\mathbf{1 0}_{H}\right)$ fields in the superpotential are restricted to the form of $W \ni \lambda_{i j} \mathbf{1 6}_{i} \mathbf{1 6}_{j} \mathbf{1 0}_{H}$. Thus, all the Yukawa matrices for up- and down-type quarks, the charged leptons, and neutrinos are the same since they all originate from the above superpotential. This is not necessarily a desired situation since the above relations result in the identical masses for the fermions in each family and no flavor mixings which are completely different from the observed structure. It is, however, possible to break the above relations naturally through the non-renormalizable interactions between the matter fields and $\mathbf{4 5}_{H}$ Higgs fields which

break $\mathrm{SO}(10)$ by its Vacuum Expectation Value (VEV) such as $\mathbf{1 6}_{i} \mathbf{1 6}_{j} \mathbf{1 0}_{H} \mathbf{4 5}{ }_{H}$, because the Yukawa interactions may feel the SO(10) breaking effect [5, 6].

However, when we take into account the fact that the Yukawa matrices have hierarchical structures and they are quite different among the quark and lepton sectors, $\mathrm{SO}(10)$ GUT has a difficulty in reproducing such structures. Many of the recent neutrino oscillation experiments support the Large Mixing Angle MSW solution [7] (LMA) to the solar neutrino anomaly $[8$, and the large mixing in the second and third generation neu- 
trinos is suggested by atmospheric neutrino oscillation experiments [9] and confirmed by the $\mathrm{K} 2 \mathrm{~K}$ long baseline experiment [10]. On the other hand, all the mixing angles in the quark sector are small and masses have hierarchical structures. The magnitude of the hierarchy is enormous, e.g., the ratio of the up-quark mass $m_{u}$ to the top-quark mass $m_{t}$ is approximately $10^{-5}$. Therefore, a mechanism to explain such a large hierarchy is necessary. The Froggatt-Nielsen (FN) mechanism is an elegant scenario to generate hierarchical structures in the Yukawa matrices [11], in which the hierarchy comes from the difference between the fundamental scale $M$ and a $\mathrm{U}(1)_{\mathrm{FN}}$ symmetry breaking scale $\left\langle\Phi_{\mathrm{FN}}\right\rangle$. The Yukawa matrices $f_{u}, f_{d}$ and $f_{e}$, and the neutrino mass matrix $m_{\nu}$ are given in terms of a small parameter $\epsilon \sim\left\langle\Phi_{\mathrm{FN}}\right\rangle / M$ and the $\mathrm{U}(1)_{\mathrm{FN}}$ charges $Q$ of the fermions by $f_{u}^{i j} \sim \epsilon^{Q\left(q_{i}\right)+Q\left(u_{j}^{c}\right)}, f_{d}^{i j} \sim \epsilon^{Q\left(q_{i}\right)+Q\left(d_{j}^{c}\right)}, f_{e}^{i j} \sim \epsilon^{Q\left(l_{i}\right)+Q\left(e_{j}^{c}\right)}$, and $m_{\nu}^{i j} \propto \epsilon^{Q\left(l_{i}\right)+Q\left(l_{j}\right)}$, where $i$ and $j$ are the generation indices, $q, u^{c}, d^{c}, l$, and $e^{c}$ represent the corresponding quarks and leptons. It is known that even if we impose the SU(5) GUT relations among the $\mathrm{U}(1)$ charges, i.e., $Q\left(q_{i}\right)=Q\left(u_{i}^{c}\right)=Q\left(e_{i}^{c}\right) \equiv Q\left(\mathbf{1 0}_{i}\right)$ and $Q\left(d_{i}^{c}\right)=Q\left(l_{i}\right) \equiv Q\left(\overline{\mathbf{5}}_{i}\right)$, we reproduce the hierarchical structures for quarks and leptons, and an appropriate neutrino mass matrix accounting for the solar neutrino anomaly by LMA. An example of the charge assignment is $Q\left(\mathbf{1 0}_{1}\right)=3, Q\left(\mathbf{1 0}_{2}\right)=2, Q\left(\mathbf{1 0}_{3}\right)=0, Q\left(\overline{\mathbf{5}}_{3}\right)=1, Q\left(\overline{\mathbf{5}}_{2}\right)=0$, and $Q\left(\overline{\mathbf{5}}_{1}\right)=0$ with $\epsilon \sim \lambda \sim 0.22$ [12, 13]. This type of Yukawa matrices is called lopsided family structure which gives successful masses and mixing patterns especially for the large mixing angles in the lepton sector [5, 12, 13, 14]. However, in SO(10) GUT, the above successful mechanism does not work in a simple way. The hierarchical structures in the fermion masses cannot be lopsided since the $\mathrm{SO}(10)$ symmetry requires $Q\left(\mathbf{1 0}_{i}\right)=Q\left(\overline{\mathbf{5}}_{i}\right)$. The situation does not change even if we introduce the non-renormalizable coupling to $\mathbf{4 5} \mathbf{5}_{H}$ to avoid the $\mathrm{SO}(10)$ relation in the fermion masses since the hierarchy is controlled by the charges of the $\mathrm{U}(1)_{\mathrm{FN}}$ symmetry, which is not related to the $\mathrm{SO}(10)$ breaking. There have been attempts to avoid this problem. An often considered way is to introduce new matter multiplets of $\mathbf{1 0}$ dimensional representation and mix or flip $d^{c}$ and $l$ in the $\mathbf{1 6}$ and those in the newly introduced 10 multiplets by the $\mathrm{SO}(10)$ breaking effect [15, 16, 17]. In the context of the six dimensional $\mathrm{SO}(10) \mathrm{GUT}$ [18, it has been also proposed the introduction of extra matter fields where the hierarchy is a consequence of the volume 
suppression factors [19]. In these scenario, $d^{c}$ and $l$ originate from the $\mathbf{1 0}$ representation fields other than 16, and thus the matter unification is spoiled. This is a generic problem in $\mathrm{SO}(10)$ GUT. Therefore, in order to solve this problem, the strong connection between the origin of flavor hierarchy and the $\mathrm{SO}(10)$ breaking is necessary.

In this sense, the recently proposed mechanism to generate the flavor hierarchy based on the wave-function profiles in the extra dimension is noteworthy [20, 21, 22, 23, 24]. In the simplest scenario, our world has an $S^{1} / Z_{2}$ compactified extra spacial dimension, and the bulk mass terms for the bulk superfields make them localize on the branes at the orbifold fixed points [22, 25]. Consequently, the values of the wave functions at a brane may be suppressed by a factor of $\exp (-|m| \pi R)$, where $m$ is the bulk mass and $R$ is the radius of the extra dimension. The flavor hierarchy is obtained in a similar way as the FN scenario such like $f^{i j} \sim \exp \left(-\left(\left|m_{i}\right|+\left|m_{j}\right|\right) \pi R\right)$. Hebecker and March-Russell considered the scenario in SU(5) GUT and showed that the hierarchy can be reproduced with a natural parameter sets [26]. Again, in $\mathrm{SO}(10)$, it seems that the $\mathrm{SO}(10)$ relation, i.e., the same $m_{i}$ for all the matter fields in each family, is too strong to reproduce the hierarchy. However, it is possible to break the $\mathrm{SO}(10)$ relations since we have additional contributions to the bulk masses m's when $\mathrm{SO}(10)$ is broken by the VEV of the bulk adjoint field whose existence is ensured by SUSY in five dimension. The additional contributions are not universal to all the matter fields but proportional to the $\mathrm{U}(1)_{X}\left(\subset \mathrm{SU}(5) \times \mathrm{U}(1)_{X} \subset\right.$ $\mathrm{SO}(10))$ charges, so that they can change the wave-function profiles without preserving the $\mathrm{SO}(10)$ relations.

In this paper, we construct an SO(10) SUSY GUT model in 5-dimensional spacetime in which the correct fermion-mass patterns are realized by the above mentioned mechanism. The matter fields in each family are unified to a single $\mathbf{1 6}$ representation field. Our scenario is compatible with already proposed doublet-triplet splitting mechanisms such as Dimopoulos-Wilczek mechanism [27], boundary condition [28, 29, etc. We also discuss a possibility of the horizontal unification which unifies the three families to the single three dimensional representation of $\mathrm{SU}(2)$ group.

This paper is organized as follows: In Section 2, we construct a model and explain the mechanism. The parameter sets to realize the suitable fermion-mass patterns are 
discussed in Section 3. Section 4 is devoted to discussions and conclusions.

\section{$2 \quad$ Model}

In this section, we first review SUSY theory on the space-time $M^{4} \times S^{1} / Z_{2}$, and show that the 5-dimensional wave function of zero mode has an exponential shape. This mechanism is a key ingredient of the fermion mass hierarchy. Then, we discuss the $\mathrm{SO}(10)$ models in this setup.

\subsection{Zero mode wave function due to bulk mass term}

We consider the $S^{1} / Z_{2}$ compactified 5-dimensional SUSY gauge theory [30]. For our purpose, it is convenient to write down the action in terms of superfields in 4-dimensional superspace 25. The action for the $\mathrm{U}(1)$ gauge theory with a bulk hypermultiplet is given by

$$
\begin{aligned}
S & =\int d^{4} x \int_{0}^{\pi R} d y\left[\int d^{2} \theta\left(\frac{1}{4} W^{\alpha} W_{\alpha}+\text { h.c. }\right)+\int d^{4} \theta\left(\partial_{y} V-\frac{1}{\sqrt{2}}(\phi+\bar{\phi})\right)^{2}\right. \\
& \left.+\int d^{4} \theta\left(H^{c} e^{2 g Q V} \bar{H}^{c}+\bar{H} e^{-2 g Q V} H\right)+\int d^{2} \theta\left(H^{c}\left(m+\partial_{y}-\sqrt{2} g Q \phi\right) H+\text { h.c. }\right)\right],
\end{aligned}
$$

where $W^{\alpha}$ and $V$ are the field strength and vector superfield associated with the $\mathrm{U}(1)$ gauge group, and $\phi$ is the chiral superfield where $A_{5}$ is in its lowest components. The gauge transformation for the superfield is $\phi \rightarrow \phi+1 /(\sqrt{2} g) \partial_{y} \Lambda$ with transformation parameter $\Lambda$. Note that the gauge coupling constant $g$ has a mass dimension of $-1 / 2$. The chiral superfields $H$ and $H^{c}$ are the components of the hypermultiplet and their $\mathrm{U}(1)$ charges are $Q$ and $-Q$, respectively ${ }^{1}$. To be invariant under the orbifold projection, the $Z_{2}$ parity is assigned to be even for $W^{\alpha}, V$, and $H$, and odd for $\phi$ and $H^{c}$. Also, the bulk mass parameter $m$ should be $Z_{2}$ odd such that $m=m \operatorname{sgn}(y)$.

By the Kaluza-Klein $(\mathrm{KK})$ decomposition, we can find the existence of a zero mode

\footnotetext{
${ }^{1}$ The superfields $H$ and $H^{c}$ do not mean the Higgs fields here.
} 
with a localized wave function. We expand the chiral superfields $H$ and $H^{c}$ as follows:

$$
H(y)=\sum_{n=0}^{\infty} H_{n}(x) f_{n}(y), \quad H^{c}(y)=\sum_{n=0}^{\infty} H_{n}^{c}(x) g_{n}(y)
$$

To obtain the normalized kinetic terms in the 4-dimensional effective action, the functions $f_{n}(y)$ and $g_{n}(y)$ are solutions of the following differential equations:

$$
\left(\frac{d}{d y}+m\right) f_{n}(y)=m_{n} g_{n}(y), \quad\left(-\frac{d}{d y}+m\right) g_{n}(y)=m_{n} f_{n}(y)
$$

with the normalization conditions:

$$
\int_{0}^{\pi R} d y f_{n}(y) f_{m}(y)=\int_{0}^{\pi R} d y g_{n}(y) g_{m}(y)=\delta_{n m} .
$$

The zero mode wave function with correct $Z_{2}$ parity is easily found to be:

$$
f_{0}(y)=\sqrt{\frac{2 m}{1-e^{-2 m \pi R}}} e^{-m y}, \quad g_{0}(y)=0 .
$$

Thus the zero mode wave function $f_{0}$ localizes exponentially at $y=0(\pi R)$ for $m>0$ $(m<0)$. For $m<0$ and $-2 m \pi R \gg 1$, since the value of the wave function at $y=0$

is exponentially suppressed by a factor of $f_{0}(0) \sim \sqrt{2|m|} e^{-|m| \pi R}$, the strength of the couplings between the bulk fields and the brane fields on the $y=0$ brane becomes weak. This effect can be the origin of the fermion mass hierarchy if the Higgs fields are confined on the brane at $y=0$ [22].

For the massive modes, the KK masses are given by

$$
m_{n}^{2}=m^{2}+\left(\frac{n}{R}\right)^{2}
$$

\section{$2.2 \quad$ Gauge symmetry breaking}

As we can see in Eq.(1), the VEV of the $\phi$ superfield is an additional contribution to the bulk mass $m$, and as a consequence the wave-function profile of the zero mode is changed [22, 25, 31. Note that the additional contribution is proportional to the $\mathrm{U}(1)$ charge. We identify the $\mathrm{U}(1)$ gauge symmetry as a subgroup of the $\mathrm{SO}(10)$ GUT gauge group and $H$ as a matter superfield of the $\mathbf{1 6}$ dimensional representation of $\mathrm{SO}(10)$, and then the different wave-function profiles are realized for the matter fields embedded in $\mathbf{1 6}$ since all the components do not have the same $\mathrm{U}(1)$ charges. 
We consider the vacuum where the $\phi$ superfield acquires the VEV while the matter superfields $H$ and $H^{c}$ do not. In order to preserve $N=1$ SUSY in the 4-dimensional effective theory (it ensures the configuration to be true vacuum), the VEVs of $F$ - and $D$-terms must vanish. The vanishing $F$-term conditions are satisfied by assuming $\langle H\rangle=$ $\left\langle H^{c}\right\rangle=0$, the $D=0$ condition is not the same as the 4-dimensional case but as follows 22, 25, 31:

$$
0=-D=\partial_{y}\langle\phi\rangle
$$

where we denote that $\phi$ is its scalar component. The contribution from the $\phi$ field is due to the 5-dimensional kinetic term, i.e., the second term in Eq.(11). The above equation is automatically satisfied if $\phi=$ const., however, as mentioned earlier, the $\phi$ field has odd parity under $y \rightarrow-y$ transformation which means that the VEV should have the form of $\langle\phi\rangle=v^{3 / 2} \operatorname{sgn}(y)$. Substituting this form in the above equation, we obtain the non-vanishing $D$-terms on the branes as follows:

$$
-D=2 v^{3 / 2}(\delta(y)-\delta(y-\pi R)) .
$$

The brane localized $D$-terms in Eq.(88) have to be canceled by the brane localized terms in the action. The most economical way is to add the Fayet-Iliopoulos $D$-terms on both branes. However, it is not applicable in our context since we identify the U(1) symmetry as a subgroup of $\mathrm{SO}(10)^{2}$. The second way is to add the brane fields charged under the U(1) symmetry. For example, if we assume the presence of chiral superfields $S_{0}$ with charge $q$ and $S_{\pi}$ with charge $-q$ which are localized on the branes at $y=0$ and $\pi R$, respectively, the $D$-term is

$$
-D=\partial_{y}\langle\phi\rangle-g q\left|\left\langle S_{0}\right\rangle\right|^{2} \delta(y)+g q\left|\left\langle S_{\pi}\right\rangle\right|^{2} \delta(y-\pi R) .
$$

The condition $D=0$ has a solution with a flat direction:

$$
2 v^{3 / 2}=g q\left|\left\langle S_{0}\right\rangle\right|^{2}=g q\left|\left\langle S_{\pi}\right\rangle\right|^{2} .
$$

This is the usual $D$-flat direction in the 4-dimensional effective theory. In the vacuum with $v \neq 0$, the $\mathrm{U}(1)$ symmetry is broken by the VEVs of $S_{0}$ and $S_{\pi}$. This is an important

\footnotetext{
${ }^{2}$ The $D$-term cancellation by the Fayet-Iliopoulos term is possible if the gauge group is broken explicitly to $\mathrm{SU}(5) \times \mathrm{U}(1)$ on the brane by the boundary conditions.
} 
point when we apply this gauge symmetry breaking to the $\mathrm{SO}(10)$ GUT breaking. The fact that the $U(1)$ symmetry is broken indicates that the $U(1)$ symmetry should not be included in the Standard Model gauge group. Moreover, the U(1) symmetry must be orthogonal to the Standard Model gauge group, otherwise the VEV of $\phi$ induces the $D$ term corresponding to the Standard Model gauge group which cannot be canceled without their breaking. Therefore, the $\mathrm{U}(1)$ subgroup of $\mathrm{SO}(10)$ is uniquely determined to be so called $\mathrm{U}(1)_{X}$ symmetry, and thus $\mathrm{SO}(10)$ is broken down to $\mathrm{SU}(5)$. As we see later, this $\mathrm{U}(1)_{X}$ breaking yields the correct masses and mixing patterns of the fermions through the wave-function deformation as well as the Majorana masses for the right-handed neutrinos.

\section{$2.3 \mathrm{SO}(10)$ model}

We construct an SO(10) GUT model which includes the above setup. The matter fields are unified to three copies of $\mathbf{1 6}$ representation, $\mathbf{1 6}_{1}, \mathbf{1 6}_{2}$, and $\mathbf{1 6}_{3}$ of $\mathrm{SO}(10)$, and they and their charge conjugation fields $\mathbf{1 6}_{i}^{c}$ can propagate into the bulk. Also, the gauge fields including a chiral superfield $\mathbf{4 5} \phi$ as the $\phi$ component in the action Eq.(11) are in the bulk. The Higgs field $\mathbf{1 0}_{H}$ is introduced as a brane field (on the $y=0$ brane) in order to obtain the suppressed Yukawa coupling constants for the first and second generations. To break $\mathrm{SO}(10) \mathrm{GUT}$, we need $\mathbf{4 5}$ Higgs field which has VEV along the $B-L$ direction in the Dimopoulos-Wilczek mechanism [27, and the Higgs fields of $\mathbf{1 6}_{H}$ and $\overline{\mathbf{1 6}}_{H}$ are necessary to reduce the rank of gauge group from five to four and give the Majorana masses to the right-handed neutrinos. We put these fields on the $y=0$ brane, and $\mathbf{1 6}_{H}^{\prime}$ and $\overline{\mathbf{1 6}}_{H}^{\prime}$ on the $y=\pi R$ brane so that they can play the same roles as $S_{0}$ and $S_{\pi}$ in the above mechanism.

In this setup, the superpotential is given by

$$
\begin{aligned}
W & =\mathbf{1 6}_{i}^{c}\left(m_{i}+\partial_{y}-\sqrt{2} g \mathbf{4 5} \phi\right) \mathbf{1 6}_{i} \\
& +\frac{\delta(y)}{M}\left[\lambda_{i j} \mathbf{1 6}_{i} \mathbf{1 6}_{j} \mathbf{1 0}_{H}+\frac{\tilde{\lambda}_{i j}}{M}\left(\mathbf{1 6}_{i} \overline{\mathbf{1 6}}_{H}\right)_{\mathbf{1}}\left(\mathbf{1 6}_{j} \overline{\mathbf{1 6}}_{H}\right)_{\mathbf{1}}\right] \\
& +\delta(y) V_{0}\left(\mathbf{4} \mathbf{5}_{H}, \mathbf{1 6}_{H}, \overline{\mathbf{1 6}}_{H}, \mathbf{1 0}_{H}, \cdots\right) \\
& +\delta(y-\pi R) V_{\pi}\left(\mathbf{1 6}_{H}^{\prime}, \overline{\mathbf{1 6}}_{H}^{\prime}\right)
\end{aligned}
$$

where $i=1,2,3$ are the generation indices and $M$ is a parameter with mass dimension one. The first term is the required form from the SUSY in five dimension. We take 
a flavor basis where the bulk mass terms $m_{i}$ are diagonalized. The Yukawa coupling constants $\lambda_{i j}$ are dimensionless quantities and the interaction terms with $\tilde{\lambda}_{i j}$ induce the Majorana masses for the right-handed neutrinos after the $\mathrm{SO}(10)$ breaking. We omit the other possible contractions which are irrelevant for the low-energy effective theory.

The potential $V_{0}$ needs to have an appropriate form for the Dimopoulos-Wilczek mechanism. According to the simplest model in Ref. [32, it is required to exist another $\mathbf{1 0}_{2 H}$, an additional pair of $\mathbf{1 6}_{2 H}$ and $\overline{\mathbf{1 6}}_{2 H}$, and several singlet fields ${ }^{3}$. On the $y=\pi R$ brane, we can write the arbitrary potential $V_{\pi}$ for the $\mathbf{1 6}_{H}^{\prime}$ and $\overline{\mathbf{1 6}}_{H}^{\prime}$, and in general, there are a number of vacua where $\mathrm{SO}(10)$ is broken. The Standard Model singlet components $N_{H}$ and $\bar{N}_{H}\left(\in \mathbf{1 6} \mathbf{6}_{H}\right.$ and $\left.\overline{\mathbf{1 6}}_{H}\right)$, and $N_{H}^{\prime}$ and $\bar{N}_{H}^{\prime}\left(\in \mathbf{1 6} \mathbf{6}_{H}^{\prime}\right.$ and $\left.\overline{\mathbf{1 6}}_{H}^{\prime}\right)$ acquire VEVs after minimizing the potential $V_{0}$ and $V_{\pi}$. Since these singlets are charged under $\mathrm{U}(1)_{X}$ subgroup of $\mathrm{SO}(10)$, there is a non-trivial condition from the $D$-flatness which is given by

$$
\begin{aligned}
0=-D_{\mathrm{U}(1)_{\mathrm{x}}}= & \delta(y)\left[v^{3 / 2}+5 g_{X}\left(\left|\left\langle N_{H}\right\rangle\right|^{2}-\left|\left\langle\bar{N}_{H}\right\rangle\right|^{2}\right)\right] \\
& -\delta(y-\pi R)\left[v^{3 / 2}-5 g_{X}\left(\left|\left\langle N_{H}^{\prime}\right\rangle\right|^{2}-\left|\left\langle\bar{N}_{H}^{\prime}\right\rangle\right|^{2}\right)\right],
\end{aligned}
$$

where $g_{X}$ is the coupling constant of the $\mathrm{U}(1)_{X}$ gauge interaction normalized such that the charge of $N_{H}$ is -5 , and $v^{3 / 2}$ is the VEV of the $\mathbf{4 5}_{\phi}$ field in the $\mathrm{U}(1)_{X}$ direction. Along the flat direction in Eq.(12), we obtain the vacuum where $v \neq 0$ as seen in the previous subsection.

Even with the potential $V_{\pi}$, there are massless modes because of the global $\mathrm{SO}(10)$ symmetry in the $y=\pi R$ sector. We assume the modes are stabilized by the SUSY breaking effect. The minimal set of the massless modes are a pair of $\mathbf{1 0}$ and $\overline{\mathbf{1 0}}$ of $\mathrm{SU}(5)$ and a Standard Model singlet since the VEVs of $N_{H}^{\prime}$ and $\bar{N}_{H}^{\prime}$ break $\mathrm{SO}(10)$ to $\mathrm{SU}(5)$, although it requires additional Higgs fields. The massless modes will obtain the masses of the order of TeV. Alternatively, the singlet mode can be stabilized without SUSY breaking effect. When we gauge the $\mathrm{U}(1)^{\prime}$ symmetry under which only $\mathbf{1 6}_{H}^{\prime}$ and $\overline{\mathbf{1 6}}_{H}^{\prime}$ are charged such as $\mathbf{1 6}_{H}^{\prime}: 1$ and $\overline{\mathbf{1 6}}_{H}^{\prime}:-1$, the constraint from the $D$-flat condition is given by

$$
\begin{aligned}
0 & =-D_{\mathrm{U}(1)^{\prime}} \\
& =\delta(y)\left[\xi_{0}+v^{\prime 3 / 2}\right]-\delta(y-\pi R)\left[-\xi_{\pi}+v^{\prime 3 / 2}-g^{\prime}\left(\left|\left\langle N_{H}^{\prime}\right\rangle\right|^{2}-\left|\left\langle\bar{N}_{H}^{\prime}\right\rangle\right|^{2}\right)\right],
\end{aligned}
$$

\footnotetext{
${ }^{3}$ It is difficult to forbid the dimension five operators which cause the proton decay in $\mathrm{SO}(10)$ model with the Dimopoulos-Wilczek mechanism. The suppression of these operators is discussed in Ref. 6, 16, 33.
} 
where $\xi_{0}$ and $\xi_{\pi}$ are the Fayet-Iliopoulos $D$-terms of the $\mathrm{U}(1)^{\prime}$ symmetry on the branes at $y=0$ and $y=\pi R$, respectively, $v^{\prime 3 / 2}$ is the VEV of the $\phi$ component associated with the $\mathrm{U}(1)^{\prime}$ symmetry, and $g^{\prime}$ is the gauge coupling constant. If $\xi_{0} \neq-\xi_{\pi}$, we obtain $\left|\left\langle N_{H}^{\prime}\right\rangle\right|^{2}-\left|\left\langle\bar{N}_{H}^{\prime}\right\rangle\right|^{2} \neq 0$, so that $v \neq 0$ is assured by Eq.(12). The singlet mode is eaten by the $\mathrm{U}(1)^{\prime}$ gauge field in this case.

In the vacuum with $v \neq 0$, the wave functions for the matter fields $\mathbf{1 0}_{i}, \overline{\mathbf{5}}_{i}$, and $\mathbf{1}_{i}$ are deformed in a different way because of the $-\sqrt{2} g \mathbf{1 6}_{i}^{c} \mathbf{4 5 _ { \phi }} \mathbf{1 6} \mathbf{6}_{i}$ terms in the superpotential (111). The bulk mass terms after the $\mathrm{SO}(10)$ breaking is modified as $m_{i} \rightarrow$ $m_{i}-\sqrt{2} g_{X} Q_{X} v^{3 / 2}$, where $Q_{X}$ is the $\mathrm{U}(1)_{X}$ charges of $\mathbf{1 0}:-1, \overline{\mathbf{5}}: 3$, and $\mathbf{1}:-5$. This is the direct connection between the origin of the flavor hierarchy and the $\mathrm{SO}(10)$ breaking. As we see in the next Section, this modification gives a realistic fermion-mass and mixing hierarchy. The violation of the unwanted SU(5) relation in the Yukawa matrices $f_{d}=f_{e}^{\mathrm{T}}$

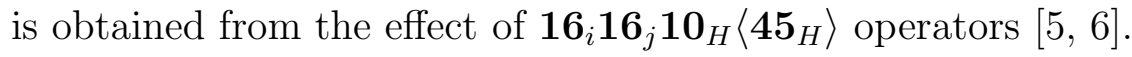

We can also consider the 5-dimensional models where the gauge symmetry is broken by the boundary conditions [28]. First, we discuss the 5-dimensional models on the spacetime $M^{4} \times S^{1} /\left(Z_{2} \times Z_{2}^{\prime}\right)$, where the $Z_{2}$ and $Z_{2}^{\prime}$ break $\mathrm{SO}(10)$ down to $\mathrm{SU}(4) \times \mathrm{SU}(2) \times$ $\mathrm{SU}(2)$ gauge symmetry and $4 \mathrm{D} N=2 \mathrm{SUSY}$ down to $N=1$, respectively. In this scenario, the $\mathrm{SO}(10)$ gauge symmetry is explicitly broken down to $\mathrm{SU}(4) \times \mathrm{SU}(2) \times \mathrm{SU}(2)$ on the brane at $y=0$ [34, 35, 36]. We do not have to worry about the doublet-triplet splitting problem since we can introduce the Higgs field as $H:(\mathbf{1}, \mathbf{2}, \mathbf{2})$ representation field and thus there is no color-triplet Higgs from the beginning [29]. The $\mathrm{SO}(10)$ breaking Higgs fields $\mathbf{1 6}_{H}$ and $\overline{\mathbf{1 6}}_{H}$ also need not to be the full multiplets and we assume the existence of $\Phi_{H}:(\overline{4}, \mathbf{1}, \mathbf{2})$ and $\bar{\Phi}_{H}:(\mathbf{4}, \mathbf{1}, \mathbf{2})$, and their VEVs break the gauge symmetry down to the Standard Model gauge group. The matter fields are also decomposed to $\Psi_{L}^{i}:(\mathbf{4}, \mathbf{2}, \mathbf{1})$ and $\Psi_{R}^{i}:(\overline{4}, 1,2)$ which are required to originate from the different 16 dimensional fields in the bulk due to the $Z_{2}$ projection. By $\mathrm{SU}(4) \times \mathrm{SU}(2) \times \mathrm{SU}(2)$ invariant but $\mathrm{SO}(10)$ non-invariant interactions, we obtain the violation of the unrealistic $\mathrm{SU}(5)$ relation of $f_{d}=f_{e}^{\mathrm{T}}$ between the Yukawa matrix of the down-type quarks $f_{d}$ and that of the charged leptons $f_{e}$. For example, the following $\mathrm{SO}(10)$ non-invariant interaction terms do not give a contribution to $f_{d}$ but to $f_{e}$ after the Standard Model singlet components of $\Phi_{H}$ and 
$\bar{\Phi}_{H}$ acquire VEVs:

$$
W \ni \frac{\delta(y)}{M} \frac{a_{i j}}{M^{2}}\left(\Psi_{L}^{i} \Phi_{H}\right)_{(\mathbf{1}, \mathbf{2}, \mathbf{2})}\left(\Psi_{R}^{j} \bar{\Phi}_{H}\right)_{(\mathbf{1}, \mathbf{1}, \mathbf{3})} H_{(\mathbf{1}, \mathbf{2}, \mathbf{2})},
$$

where $a_{i j}$ are the dimensionless coupling constants. Including these contributions, we do not have any relation between the down-type-quark and lepton Yukawa matrices. Since the contributions should be comparable to the $\mathrm{SO}(10)$ invariant terms of $\Psi_{L} \Psi_{R} H$ in order to reproduce the fermion masses, the parameter $M$ is required to be the same scale as the VEV of $\Phi_{H}$. Therefore, we take all the dimensionfull parameters to be of the order of the GUT scale $\sim 10^{16} \mathrm{GeV}$.

In addition, we can discuss the scenario where the $Z_{2}$ and $Z_{2}^{\prime}$ break $4 \mathrm{D} N=2 \mathrm{SUSY}$ down to $N=1$ and $\mathrm{SO}(10)$ down to $\mathrm{SU}(5) \times \mathrm{U}(1)_{X}$, respectively. Because of the $Z_{2}^{\prime}$

projection, we introduce six copies of $\mathbf{1 6}$ fields in the bulk. In this model, $\mathbf{1 0}_{i}$ and $\left(\overline{\mathbf{5}}_{i}\right.$ and $\mathbf{1}_{i}$ ) come from the different $\mathbf{1 6}$ 's, and then, can have different bulk masses. So, similar to SU(5) models in Ref. [26], one can generate the fermion-mass hierarchy by choosing a parameter set $m_{i}(i=1-6)$ for six 16 representation matter fields. The doublet-triplet splitting problem can be solved by Dimopoulos-Wilczek mechanism. Essentially speaking, this is an $\mathrm{SU}(5)$ model but not $\mathrm{SO}(10)$ model, and then the matter unification is spoiled. Thus, we will not discuss it in this paper.

\section{Fermion masses}

We consider the fermion masses in this scenario with the assumption that all the components of the Yukawa matrices on the brane are of order unity. We also assume that neither $\mathrm{SO}(10)$ nor $\mathrm{SU}(5)$ relations among the Yukawa matrices maintain because of the symmetry breaking effects on the brane as discussed in Subsection 2.3. The hierarchical structures in the observed fermion masses and mixings are realized by means of the wave-function profiles of the zero modes discussed in Subsection 2.1. The lopsided family structure is given through the effect of non-vanishing $v^{3 / 2}=\left\langle\mathbf{4} \mathbf{5}_{\phi}\right\rangle$ in the bulk.

The observed hierarchy can be approximately expressed by the power of the Cabibbo 
angle $\lambda \sim 0.22$. The CKM matrix is given by

$$
V_{\mathrm{CKM}} \sim\left(\begin{array}{ccc}
1 & \lambda & \lambda^{3} \\
-\lambda & 1 & \lambda^{2} \\
-\lambda^{3} & -\lambda^{2} & 1
\end{array}\right)
$$

The ratios of the masses at the GUT scale 37] are given by

$$
\begin{aligned}
& m_{u}: m_{c}: m_{t} \sim \lambda^{7}: \lambda^{4}: 1, \\
& m_{d}: m_{s}: m_{b} \sim \lambda^{4}: \lambda^{2}: 1, \\
& m_{e}: m_{\mu}: m_{\tau} \sim \lambda^{5}: \lambda^{2}: 1 .
\end{aligned}
$$

Also, the ratio of the neutrino masses in the second and third generations is estimated from the ratio of the $\Delta m^{2}$ of the solar and atmospheric neutrino oscillations. If we assume the hierarchical mass pattern, the ratio is given by

$$
m_{\nu_{2}}: m_{\nu_{3}} \sim \sqrt{\frac{\Delta m_{\mathrm{solar}}^{2}}{\Delta m_{\mathrm{atm} .}^{2}}} \sim \lambda^{1-2}: 1,
$$

where we used the LMA solution for the solar neutrino oscillation which requires nearly bimaximal mixing in the Maki-Nakagawa-Sakata (MNS) matrix [38] as follows:

$$
V_{\mathrm{MNS}} \sim\left(\begin{array}{ccc}
1 / \sqrt{2} & -1 / \sqrt{2} & \epsilon \\
1 / 2 & 1 / 2 & -1 / \sqrt{2} \\
1 / 2 & 1 / 2 & 1 / \sqrt{2}
\end{array}\right)
$$

where $\epsilon$ is a small parameter.

The Yukawa couplings in the effective theory are the products of the Yukawa couplings and the values of the wave functions on the brane at $y=0\left(f_{0}(0)\right)$. From Eq.(15), the $f_{0}(0)$ 's are given by

$$
f_{0}(0)^{i,(r)}=\sqrt{\frac{2|M| a_{i}^{(r)}}{1-e^{-2 a_{i}^{(r)} c}}} \sim\left\{\begin{array}{ll}
\sqrt{2\left|M a_{i}^{(r)}\right|} e^{-\left|a_{i}^{(r)}\right| c} & \left(a_{i}^{(r)}<0\right) \\
\sqrt{2|M| a_{i}^{(r)}} & \left(a_{i}^{(r)}>0\right) \\
\sqrt{|M| / c} & \left(a_{i}^{(r)}=0\right)
\end{array},\right.
$$

where the superscript $r$ stands for the belonging $\mathrm{SU}(5)$ representations such as $\mathbf{1 0}, \overline{\mathbf{5}}$, and $\mathbf{1}$, and $i$ is the generation index. The parameter $c$ is $|M| \pi R$. The $a_{i}^{(r)}$ parameters 
are dimensionless quantities expressed in terms of $\mu_{i} \equiv m_{i} /|M|$ and $k \equiv g_{X} v^{3 / 2} /|M|$ as follows: ${ }^{4}$

$$
a_{i}^{(\mathbf{1 0})}=\mu_{i}+k, \quad a_{i}^{(\overline{\mathbf{5}})}=\mu_{i}-3 k, \quad a_{i}^{(\mathbf{1})}=\mu_{i}+5 k .
$$

The parameter $k$ represents the effect of $\mathrm{SO}(10)$ breaking and the $\mathrm{SO}(10)$ relation of $a_{i}^{(\mathbf{1 0})}=a_{i}^{(\overline{\mathbf{5}})}=a_{i}^{(\mathbf{1})}$ restores in the limit of $k \rightarrow 0$. This parameter plays an important role to reproduce the fermion-mass hierarchy. Hereafter, we take $\left|\mu_{3}\right|=1$ which can be done without loss of generality. Defining the dimensionless suppression factors $n_{i}^{(R)} \equiv$ $f_{0}(0)^{i,(r)} / \sqrt{|M|}$, we obtain the formulae of the Yukawa matrices:

$$
f_{u}^{i j}=\lambda_{u}^{i j} n_{i}^{(\mathbf{1 0})} n_{j}^{(\mathbf{1 0})}, \quad f_{d}^{i j}=\lambda_{d}^{i j} n_{i}^{(\mathbf{1 0})} n_{j}^{(\overline{\mathbf{5}})}, \quad f_{e}^{i j}=\lambda_{e}^{i j} n_{i}^{(\overline{\mathbf{5}})} n_{j}^{(\mathbf{1 0})} .
$$

The matrices $\lambda_{u}, \lambda_{d}$, and $\lambda_{e}$ are the Yukawa matrices on the brane at $y=0$ and their components are of order unity. For neutrinos, the Yukawa matrix and the Majorana mass matrix for the right-handed neutrinos are given by

$$
f_{\nu}^{i j}=\lambda_{\nu}^{i j} n_{i}^{(\overline{\mathbf{5}})} n_{j}^{(\mathbf{1})}, \quad M_{R}^{i j}=\widetilde{M}_{R}^{i j} n_{i}^{(\mathbf{1})} n_{j}^{(\mathbf{1})} .
$$

Again $\lambda_{\nu}$ and $\widetilde{M}_{R}$ are the Yukawa matrix and the Majorana mass matrix $\left(\widetilde{M}_{R}=\tilde{\lambda}\left\langle\overline{\mathbf{1 6}}_{H}\right\rangle^{2} / M\right)$ on the brane at $y=0$, respectively. Since the neutrino masses are given through the seesaw mechanism $m_{\nu}=f_{\nu} M_{R}^{-1} f_{\nu}^{\mathrm{T}}$ [39], the suppression factors of $n_{i}^{(\mathbf{1})}$ cancel in this formula and thus the neutrino mass matrix is expressed by

$$
m_{\nu}^{i j}=\left(\lambda_{\nu} \widetilde{M}_{R}^{-1} \lambda_{\nu}^{\mathrm{T}}\right)^{i j} n_{i}^{(\overline{\mathbf{5}})} n_{j}^{(\overline{\mathbf{5}})} v_{2}^{2}
$$

where $v_{2} \sim 174 \mathrm{GeV}$ is the VEV of the Higgs field which couples to the up-type quarks and neutrinos.

The formulae in Eqs.(23) and (24) are the same as those of the FN mechanism except the relations in Eq.(22). The number of the free parameters is reduced to be four which is $\mu_{1}, \mu_{2}, c$, and $k$ compared to the case of FN mechanism in which six charges (three $Q\left(\mathbf{1 0}_{i}\right)$ and three $\left.Q\left(\overline{\mathbf{5}}_{i}\right)\right)$ and a parameter $\epsilon=\langle\Phi\rangle / M$ are adjustable. Interestingly, we see in the following that the four parameters are sufficient to reproduce the Yukawa structures.

\footnotetext{
${ }^{4}$ In the case where $\mathrm{SO}(10)$ is broken down to $\mathrm{SU}(4) \times \mathrm{SU}(2) \times \mathrm{SU}(2)$ by the boundary condition as discussed in the previous section, the left-handed multiplet $\Psi_{L}^{i}$ and right-handed one $\Psi_{R}^{i}$ may have different bulk masses $m_{i}^{L}$ and $m_{i}^{R}$. However, as we see later, only the case with $m_{i}^{L} \sim m_{i}^{R}$ gives the correct mass patterns, and this is suitable to the horizontal unification and the gauge coupling unification.
} 
Our goal is to reproduce the lopsided family structures given as follows:

$$
f_{u} \sim\left(\begin{array}{ccc}
\lambda^{6} & \lambda^{5} & \lambda^{3} \\
\lambda^{5} & \lambda^{4} & \lambda^{2} \\
\lambda^{3} & \lambda^{2} & 1
\end{array}\right), \quad f_{d} \sim f_{e}^{\mathrm{T}} \sim\left(\begin{array}{ccc}
\lambda^{4} & \lambda^{3} & \lambda^{3} \\
\lambda^{3} & \lambda^{2} & \lambda^{2} \\
\lambda & 1 & 1
\end{array}\right), \quad m_{\nu} \propto\left(\begin{array}{ccc}
\lambda^{2} & \lambda & \lambda \\
\lambda & 1 & 1 \\
\lambda & 1 & 1
\end{array}\right) .
$$

We can see that this form of Yukawa matrices reproduce the hierarchical structures in Eqs.(15)-(20) when we take into account the $O(1)$ ambiguities. The large mixing in the 1-2 generation of the neutrinos is realized when the determinant of the 2-3 submatrix in $m_{\nu}$ is $O(\lambda)$, which is also consistent with the mass relation in Eq.(19). As mentioned in Introduction, the form is suitable to the FN mechanism. Similarly, in our mechanism, the above structures are given when the suppression factors have the form of

$$
n_{i}^{(\mathbf{1 0})} \sim\left(\lambda^{3}, \lambda^{2}, 1\right), \quad n_{i}^{(\overline{\mathbf{5}})} \sim(\lambda, 1,1) .
$$

The above type of suppression factors is obtained in the following way. First, we consider the $n_{i}^{(\mathbf{1 0})}$ factors. The last component in $n_{i}^{(\mathbf{1 0})}$ of $O(1)$ is given if $\mathbf{1 0}_{3}$ is localized on the $y=0$ brane. From Eq.(21), this is achieved by

$$
a_{3}^{(\mathbf{1 0})}= \pm 1+k \geq 0
$$

where \pm represent the undetermined sign of $\mu_{3}$. The second component in $n_{i}^{(\mathbf{1 0})}$ of $O\left(\lambda^{2}\right)$ is, in contrast, given by the $\mathbf{1 0}_{2}$ localization on the $y=\pi R$ brane such that

$$
a_{2}^{(\mathbf{1 0})} c=\left(\mu_{2}+k\right) c \sim \log \lambda^{2} \sim-3.0
$$

The first component in $n_{i}^{(\mathbf{1 0})}$ of $O\left(\lambda^{3}\right)$ determine the relative size of the $\mu_{1}$ parameter as follows:

$$
\left(a_{1}^{(\mathbf{1 0})}-a_{2}^{(\mathbf{1 0})}\right) c=\left(\mu_{1}-\mu_{2}\right) c \sim \log \frac{O\left(\lambda^{3}\right)}{O\left(\lambda^{2}\right)} \sim-1.5 .
$$

Next, the $n_{i}^{(\overline{\mathbf{5}})}$ factors constrain the parameters. The second and last components in $n_{i}^{(\overline{\mathbf{5}})}$ of $O(1)$ give

$$
a_{3}^{(\overline{\mathbf{5}})}= \pm 1-3 k \geq 0, \quad a_{2}^{(\overline{\mathbf{5}})}=\mu_{2}-3 k \geq 0 .
$$

The suppression of the first component in $n_{i}^{(\overline{\mathbf{5}})}$ is given by

$$
a_{1}^{(\overline{\mathbf{5}})}=\left(\mu_{1}-3 k\right) c \sim \log \lambda \sim-1.5 .
$$




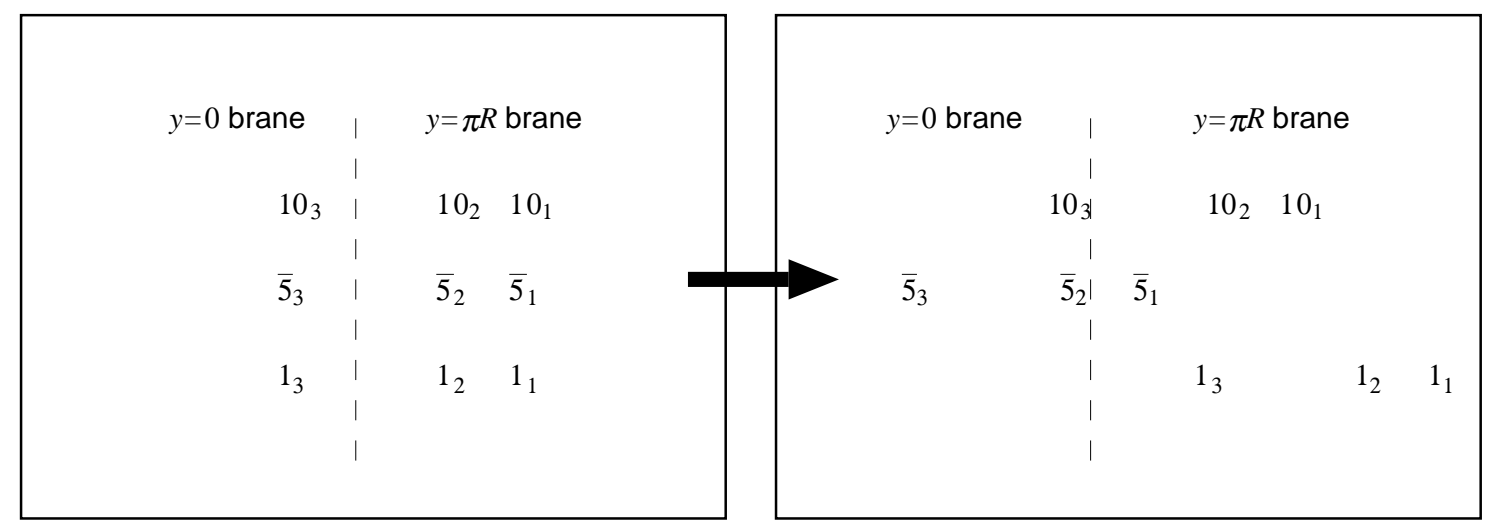

Figure 1: The configurations of the matter fields before and after the $\mathrm{SO}(10)$ breaking are shown.

From the above conditions, the sign of $\mu_{3}$ is determined to be $\mu_{3}=+1$ and the $k c$ parameter is approximately given by

$$
k c \sim-0.75
$$

Once we postulate the value of $k$ or $c$, we can determine the values of $\mu_{1}$ and $\mu_{2}$ by Eqs.(29) and (30). For example, for $k \sim-0.75$ and $c \sim 1$, we find $\mu_{1} \sim-3.75$ and $\mu_{2} \sim-2.25$. We schematically explain this mechanism in Fig 1. Without $\mathrm{SO}(10)$ breaking, $\mathbf{1 0}_{i}$ and $\overline{\mathbf{5}}_{i}$, of course, have the same profiles. However, once the $\mathrm{SO}(10)$ breaking effect is turned on, the profiles are deformed in such a way that the Yukawa structures become the currently observed form.

When we think about the origin of the bulk mass parameters $m_{i}$, there is a possibility that the origin is the spontaneous breaking of an $\mathrm{SU}(2)$ horizontal symmetry ${ }^{5}$. In fact, the suppression of the $n_{1}^{(\overline{\mathbf{5}})}$ is not necessarily important. Even if $n_{1}^{(\overline{\mathbf{5}})}=O(1)$, the Yukawa matrices are in a good shape if we take into account the $O(1)$ ambiguities in the Yukawa couplings on the brane. The neutrino masses and mixings are explained by the anarchy scenario [40]. In that scenario, the form of the suppression factors is given by [41]

$$
n_{i}^{(\mathbf{1 0})} \sim\left(\lambda^{4}, \lambda^{2}, 1\right), \quad n_{i}^{(\overline{5})} \sim(1,1,1) .
$$

Although this type of suppression factors gives a small value $\sim \lambda^{2}$ to the Cabibbo angle, we think that the observed large value of the Cabibbo angle is due to the $O(1)$ ambiguity.

\footnotetext{
${ }^{5}$ Similarly, one can consider the U(1) flavor symmetry.
} 
An interesting possibility arises in this case. We find a parameter set of $k \sim-1, c \sim 3$, $\mu_{1} \sim-1, \mu_{2} \sim 0$, and $\mu_{3}=1$, which gives above suppression factors. With the relation $\mu_{1}=-\mu_{3}$ and $\mu_{2}=0$, there is a possibility that the origin of the bulk mass parameters $m_{i}$ are the VEV of the bulk adjoint field of the $\mathrm{SU}(2)$ horizontal symmetry in which the three generations of the $\mathbf{1 6}$ representation matter fields are unified to $\mathbf{3}$ representation. As discussed in the previous section, the VEV of the bulk $\mathrm{SU}(2)$ adjoint field gives rise to the $D$-terms on the branes and they are canceled by the $D$-terms given by the VEVs of the localized fields in the fundamental representation on the branes. The VEVs break the $\mathrm{SU}(2)$ symmetry completely and the couplings to the matter fields may induce off diagonal components of the Yukawa couplings which are absent in the $\mathrm{SU}(2)$ symmetric limit. This possibility is a special feature in $\mathrm{SO}(10)$ GUT and can not be realized in $\mathrm{SU}(5)$ GUT. The existence of the $\mathrm{U}(1)_{X}$ breaking is essential to give the different bulk masses to $\mathbf{1 0}$ and $\overline{\mathbf{5}}$ fields.

Finally, we comment on the neutrino masses. If we assume that all the dimensionfull parameters are of the order of $10^{16} \mathrm{GeV}$, we find the mass of the heaviest neutrino in Eq.(25) is one order of magnitude smaller than the favored value by the atmospheric neutrino data of $\sim 0.05 \mathrm{eV}$. However, this is not a serious problem because the $O(1)$ ambiguities easily reduce the Majorana masses of the right-handed neutrinos and/or increase the Dirac neutrino Yukawa couplings.

Although the right-handed neutrino masses do not affect the neutrino masses, they might be important from the viewpoint of leptogenesis [42. In the scenario with Eq.(33), our predictions on the lightest right-handed neutrino masses are given by

$$
M_{R}^{1} \sim M_{R}^{11} \sim 10^{-7} M \sim 10^{9} \mathrm{GeV}
$$

This value is compatible with the leptogenesis scenario [13, 43]. Of particular interest is the case with the horizontal symmetry. With the values of $k \sim-1$ and $c \sim 3$, we obtain the right-handed neutrino masses $M_{R}^{1} \sim 1-10 \mathrm{GeV}, M_{R}^{2} \sim 100-1000 \mathrm{GeV}$, and $M_{R}^{3} \sim 10^{5-6} \mathrm{GeV}$. Since the lightest right-handed neutrino does not enter the thermal equilibrium before the electroweak phase transition, the partial lepton asymmetry carried by the particles except for the right-handed neutrino is converted to the baryon number by the sphaleron process even if there is no net $B-L$ asymmetry [4]. 
We assumed in the superpotential in Eq.(11) that the right-handed neutrinos acquire masses from the VEVs of $y=0$ brane fields. Alternatively, we can change the source of their masses to the $y=\pi R$ brane. However, in this case, Eq.(25) is not applied, and then it leads too small neutrino masses or non-suitable forms for the LMA solution.

\section{Discussions and Conclusions}

We consider the scenarios with the lopsided family structure in SO(10) GUT by using the 5 -dimensional wave function profile. We can reproduce the fermion masses and mixings including the LMA solution without introducing extra matter fields. The key point is that we directly connect the $\mathrm{SO}(10)$ breaking effect to the origin of fermion-mass hierarchy, which is difficult in the usual FN scenario.

We used the bulk mass terms as a origin of the hierarchy. The $\mathrm{SO}(10)$ breaking effect gives additional contributions to the bulk mass terms so that the Yukawa matrices do not obey the unrealistic $\mathrm{SO}(10)$ relation $f_{u} \sim f_{d} \sim f_{e} \sim f_{\nu}$. Moreover, we find the possibility of $\mathrm{SU}(2)$ horizontal unification of three generations, and that is possible in $\mathrm{SO}(10)$ rather than SU(5) GUT.

"SU(5) or $\mathrm{SO}(10)$ ?" is an interesting question. From the viewpoint of the doublettriplet splitting problem, $\mathrm{SO}(10)$ might have an advantage since it has an intrinsic possibility to realize the doublet-triplet splitting by the Dimopoulos-Wilczek mechanism, but it is not easy. Since we need to reduce the rank of gauge group, the Higgs sector becomes complicated. It is, therefore, difficult to judge from this viewpoint. The fermion-mass hierarchy is another interesting viewpoint. We can say that $\mathrm{SU}(5)$ is better than $\mathrm{SO}(10)$ in this case. The $\mathrm{SU}(5)$ relation of $f_{d} \sim f_{e}^{\mathrm{T}}$ is approximately good and the hierarchy is compatible to the FN mechanism. On the other hand, $\mathrm{SO}(10)$ symmetry is too strong and it is required to introduce extra-matter fields. However, the concept of the matter unification is too beautiful to be thrown away. In this sense, the wave-function localization is an attractive possibility which enables a matter unified $\mathrm{SO}(10)$ GUT to be realistic and opens up a further possibility of horizontal unification.

Considering the SUSY breaking in this scenario is also interesting. Severe experimental limits of the processes with Flavor Changing Neutral Current (FCNC) and CP violation 
restrict the sfermion mass matrices to be some specific forms. In our scenario, there is an interesting possibility if SUSY is broken on the $y=\pi R$ brane. Because of the wavefunction profiles, only the first and second generation fields can feel the SUSY breaking effect strongly. This is the desired situation for the effective SUSY scenario [45] in which the sfermions of the first and second generations have large soft masses of the order of $10 \mathrm{TeV}$ so as to avoid the constraints from the FCNC and CP violating processes, and the third generation sfermions are as light as $\mathrm{O}(100) \mathrm{GeV}$ for naturalness. Consideration in this direction is interesting, although we need the mechanism to suppress the gaugino masses and to generate the $\mu$-term. The gauge mediation [46] is another candidate where the $\mathbf{1 6}_{H}^{\prime}$ and $\overline{\mathbf{1 6}}_{\mathbf{H}}^{\prime}$ fields on the $y=\pi R$ brane may be the messenger fields.

\section{Acknowledgments}

We would like to thank Hooman Davoudiasl, Carlos Pena-Garay, and Scott Thomas for useful discussions. The work of R.K. was supported by DOE grant DE-FG02-90ER40542. The work of T.L. was supported by the National Science Foundation under Grant No. PHY0070928.

\section{References}

[1] E. Witten, Nucl. Phys. B 188, 513 (1981);

S. Dimopoulos, S. Raby and F. Wilczek, Phys. Rev. D 24, 1681 (1981);

S. Dimopoulos and H. Georgi, Nucl. Phys. B 193, 150 (1981);

N. Sakai, Z. Phys. C 11, 153 (1981).

[2] See, for example, H. P. Nilles, Phys. Rept. 110, 1 (1984).

[3] H. Georgi and S. L. Glashow, Phys. Rev. Lett. 32, 438 (1974).

[4] C. Giunti, C. W. Kim and U. W. Lee, Mod. Phys. Lett. A 6, 1745 (1991);

U. Amaldi, W. de Boer and H. Furstenau, Phys. Lett. B 260, 447 (1991);

P. Langacker and M. x. Luo, Phys. Rev. D 44, 817 (1991).

[5] C. H. Albright, K. S. Babu and S. M. Barr, Phys. Rev. Lett. 81, 1167 (1998).

[6] K. S. Babu, J. C. Pati and F. Wilczek, Nucl. Phys. B 566, 33 (2000). 
[7] L. Wolfenstein, Phys. Rev. D 17, 2369 (1978);

S. P. Mikheev and A. Y. Smirnov, Sov. J. Nucl. Phys. 42, 913 (1985) [Yad. Fiz. 42, 1441 (1985)]; Nuovo Cim. C 9, 17 (1986).

[8] S. Fukuda et al. [Super-Kamiokande Collaboration], Phys. Rev. Lett. 86, 5656 (2001); Q. R. Ahmad et al. [SNO Collaboration], Phys. Rev. Lett. 89, 011301 (2002); Phys. Rev. Lett. 89, 011302 (2002);

K. Eguchi et al. [KamLAND Collaboration], Phys. Rev. Lett. 90, 021802 (2003).

[9] Y. Fukuda et al. [Super-Kamiokande Collaboration], Phys. Rev. Lett. 81, 1562 (1998).

[10] M. H. Ahn et al. [K2K Collaboration], arXiv:hep-ex/0212007.

[11] C. D. Froggatt and H. B. Nielsen, Nucl. Phys. B 147, 277 (1979).

[12] J. Sato and T. Yanagida, Phys. Lett. B 430, 127 (1998);

T. Yanagida and J. Sato, Nucl. Phys. Proc. Suppl. 77, 293 (1999).

[13] W. Buchmuller and T. Yanagida, Phys. Lett. B 445, 399 (1999).

[14] N. Irges, S. Lavignac and P. Ramond, Phys. Rev. D 58, 035003 (1998).

[15] Y. Nomura and T. Yanagida, Phys. Rev. D 59, 017303 (1999);

Y. Nomura and T. Sugimoto, Phys. Rev. D 61, 093003 (2000);

C. H. Albright and S. M. Barr, Phys. Rev. Lett. 85, 244 (2000).

[16] N. Maekawa, Prog. Theor. Phys. 106, 401 (2001).

[17] R. Kitano and Y. Mimura, Phys. Rev. D 63, 016008 (2001).

[18] T. Asaka, W. Buchmuller and L. Covi, Phys. Lett. B 523, 199 (2001);

L. J. Hall, Y. Nomura, T. Okui and D. R. Smith, Phys. Rev. D 65, 035008 (2002);

T. Li, Nucl. Phys. B 619, 75 (2001);

B. Kyae and Q. Shafi, arXiv:hep-ph/0212331.

[19] N. Haba and Y. Shimizu, arXiv:hep-ph/0210146;

N. Haba, T. Kondo and Y. Shimizu, Phys. Lett. B 535, 271 (2002); Phys. Lett. B 531, 245 (2002).

[20] N. Arkani-Hamed and M. Schmaltz, Phys. Rev. D 61, 033005 (2000).

[21] E. A. Mirabelli and M. Schmaltz, Phys. Rev. D 61, 113011 (2000).

[22] D. E. Kaplan and T. M. Tait, JHEP 0111, 051 (2001). 
[23] M. Kakizaki and M. Yamaguchi, arXiv:hep-ph/0110266.

[24] N. Haba and N. Maru, Phys. Rev. D 66, 055005 (2002).

[25] N. Arkani-Hamed, T. Gregoire and J. Wacker, JHEP 0203, 055 (2002).

[26] A. Hebecker and J. March-Russell, Phys. Lett. B 541, 338 (2002).

[27] S. Dimopoulos and F. Wilczek, NSF-ITP-82-07.

[28] Y. Kawamura, Prog. Theor. Phys. 105, 999 (2001);

G. Altarelli and F. Feruglio, Phys. Lett. B 511, 257 (2001);

L. J. Hall and Y. Nomura, Phys. Rev. D 64, 055003 (2001).

[29] A. Hebecker and J. March-Russell, Nucl. Phys. B 613, 3 (2001).

[30] A. Pomarol and M. Quiros, Phys. Lett. B 438, 255 (1998).

[31] R. Barbieri, R. Contino, P. Creminelli, R. Rattazzi and C. A. Scrucca, Phys. Rev. D 66, 024025 (2002);

S. Groot Nibbelink, H. P. Nilles and M. Olechowski, Phys. Lett. B 536, 270 (2002);

Nucl. Phys. B 640, 171 (2002);

H. Abe, T. Higaki and T. Kobayashi, arXiv:hep-th/0210025

[32] S. M. Barr and S. Raby, Phys. Rev. Lett. 79, 4748 (1997).

[33] K. S. Babu and S. M. Barr, Phys. Rev. D 48, 5354 (1993);

J. C. Pati, arXiv:hep-ph/0204240.

[34] R. Dermisek and A. Mafi, Phys. Rev. D 65, 055002 (2002).

[35] C. H. Albright and S. M. Barr, Phys. Rev. D 67, 013002 (2003).

[36] H. D. Kim and S. Raby, JHEP 0301, 056 (2003).

[37] H. Fusaoka and Y. Koide, Phys. Rev. D 57, 3986 (1998).

[38] Z. Maki, M. Nakagawa and S. Sakata, Prog. Theor. Phys. 28, 870 (1962).

[39] T. Yanagida, in Proceedings of the Workshop on Unified Theory and Baryon Number of the Universe, eds. O. Sawada and A. Sugamoto (KEK, 1979) p.95;

M. Gell-Mann, P. Ramond, and R. Slansky, in Supergravity, eds. P. van Nieuwenhuizen and D. Freedman (North Holland, Amsterdam, 1979).

[40] L. J. Hall, H. Murayama and N. Weiner, Phys. Rev. Lett. 84, 2572 (2000);

N. Haba and H. Murayama, Phys. Rev. D 63, 053010 (2001);

A. de Gouvea and H. Murayama, arXiv:hep-ph/0301050. 
[41] J. Hisano, K. Kurosawa and Y. Nomura, Nucl. Phys. B 584, 3 (2000).

[42] M. Fukugita and T. Yanagida, Phys. Lett. B 174, 45 (1986).

[43] W. Buchmuller and M. Plumacher, Phys. Lett. B 389, 73 (1996).

[44] K. Dick, M. Lindner, M. Ratz and D. Wright, Phys. Rev. Lett. 84, 4039 (2000); H. Murayama and A. Pierce, Phys. Rev. Lett. 89, 271601 (2002).

[45] M. Dine, A. Kagan and S. Samuel, Phys. Lett. B 243, 250 (1990);

S. Dimopoulos and G. F. Giudice, Phys. Lett. B 357, 573 (1995);

A. Pomarol and D. Tommasini, Nucl. Phys. B 466, 3 (1996);

A. G. Cohen, D. B. Kaplan and A. E. Nelson, Phys. Lett. B 388, 588 (1996).

[46] M. Dine and A. E. Nelson, Phys. Rev. D 48, 1277 (1993);

M. Dine, A. E. Nelson and Y. Shirman, Phys. Rev. D 51, 1362 (1995);

M. Dine, A. E. Nelson, Y. Nir and Y. Shirman, Phys. Rev. D 53, 2658 (1996). 